\title{
A Direct Method for Linear Dynamical Problems in Continuum Mechanics with Random Loads*
}

\author{
By Frederic Y. M. Wan
}

\section{Introduction}

Linear dynamical problems in continuum mechanics are usually formulated as initial-boundary value problems in linear partial differential equations. In general, we have a vector equation

$$
u_{t}+L[u]=f(x, t) \quad(t>0, x \in V)
$$

for the unknown column vector $\mathrm{u}(\mathrm{x}, \mathrm{t})$ defined in some volume in space, V. Here, $L$ is a linear partial differential operator in the spatial coordinate(s), $x_{k}$, though the known continuous coefficients may be functions of $t$ as well. For example, in a simple model for a single lifting rotor blade in forward flight, the dimensionless transverse displacement of the uniform blade satisfies the dimensionless equation

$$
\begin{aligned}
& \zeta^{4} w_{x x x x}-\frac{1}{2}\left(1-x^{2}\right) w_{x x}+\left(x+\gamma_{0} \mu \cos t|x+\mu \sin t|\right) w_{x} \\
& \quad+\left(\beta+\gamma_{0}|x+\mu \sin t|\right) w_{t}+w_{t t}=\gamma_{0}|x+\mu \sin t| N(x, t)
\end{aligned}
$$

for $t>0$ and $0<x<1$, where $\zeta, \beta, \gamma_{0}$ and $\mu$ are known parameters and $N(x, t)$ is a random function with known statistics. By setting $\boldsymbol{u}_{\mathbf{1}}=\mathrm{w}$ and $\boldsymbol{u}_{\mathbf{2}}=\boldsymbol{w}_{\boldsymbol{t}}$, equation (1.2) can be written in the form of (1.1) with coefficients of the operator $L$ being continuous functions of both $\boldsymbol{x}$ and $\boldsymbol{t}$.

Equation (1.1) is supplemented by some initial condition at $t=0$ and some boundary condition(s) on the boundary $S$ of $V$. For simplicity, we take these to be the homogenous conditions

$$
u(x, 0)=0 \quad(x \in \bar{V})
$$

and

$$
B[u]=0 \quad(t>0, x \in S)
$$

where $B$ is in general a linear partial differential operator in $\underline{x}_{k}$ whose continuous coefficients may be functions of both $x$ and $t$, and where $\bar{V}^{k}$ is the closure of $V$, $V$ and its boundary $S$.

* Presented at the XIIIth International Congress of Theoretical and Applied Mechanics, Moscow, August, 1972. 
If $f(x, t)$ is a known vector function, the solution of this problem can be written down immediately in terms of the associated Green's function

$$
u(x, t)=\int_{V} \int_{0}^{t} G\left(x, t: x^{\prime}, t^{\prime}\right) f\left(x^{\prime}, t^{\prime}\right) d t^{\prime} d x^{\prime} .
$$

When the load term, $f(x, t)$, is a random function of known statistics, a solution of the problem consists of obtaining moment functions (or joint probability density functions) of all orders of the response $u(x, t)$. With the help of (1.5) this is in principle straightforward. For example, the expected value (first moment function) of $u$ is given by

$$
\left.\langle u(x, t)\rangle=\int_{v} \int_{0}^{t} \mathrm{G}\left(\mathrm{x}, \boldsymbol{t} \mid \mathrm{x}^{\prime}, \mathrm{t}^{\prime}\right)<f\left(x^{\prime}, t^{\prime}\right)\right\rangle d t^{\prime} d x^{\prime}
$$

where $\langle\ldots\rangle$ is the ensemble-averaging operation. The higher order moments can be determined by forming the ensemble average of different combinations of $\mathrm{u}(\mathrm{x}, \mathrm{t})$ with the help of (1.5).

Unfortunately, the Green's function, $\mathrm{G}\left(\mathrm{x}, \mathrm{t} \mid \mathrm{x}^{\prime}, \mathrm{t}^{\prime}\right)$, of (1.1) (1.3) and (1.4) can not be obtained in terms of elementary or special functions except for the simplest cases. In general, we will have to obtain $\mathrm{G}$ by some numerical method, the desired statistics of the response will still have to be calculated by multiple integration. Even if we are willing to settle for the mean and the mean square value of $u$, the above procedure (henceforth referred to as the Green's function method) is still impractical since it requires an enormous amount of machine computation even for a spatially one-dimensional problem such as the rotor blade problem.

More efficient methods than the Green's function method are available for the first and second order statistics (which are all we need for a Gaussian process) of certain special classes of problems. For example, the normal mode approach and the transfer matrix approach $[1,2]$ have been used successfully for many problems in structural mechanics. To varying degrees, these methods depend on special properties of the relevant differential operators such as constant coefficients, self-adjointness, separability with respect to the independent variables, etc. On the other hand, operators which do not have any of these nice properties do occur in the analysis of technical problems. For instance, none of these efficient methods applies to equation (1.2) unless we are willing to make some approximation which may or may not be justified.

To seek a more effective method for the first and second order statistics of $u$ for non-self-adjoint, non-separable equations with variable coefficients such as (1.2), we were motivated by a "direct method" described for linear time invariant discrete dynamical systems in [1] (pp. 150-152) and references therein, and used recently for time-varying systems in [3,4]. The essential feature of that method is to formulate (nonstochastic) initial value problems for the mean, the covariance matrix and the correlation matrix. * At the very least, this approach enables us to obtain the desired statistics by numerical integration in a straightforward manner and the necessary calculation is much more manageable than working directly

* Though the method can be used for higher order statistics, only first and second order statistics are calculated in practice. 
with the equivalence of (1 .5) whenever the impulse response function of the discrete dynamical system can not be found in terms of known functions [4].

To extend this "direct method" to problems for continuous media, we find, by ensemble-averaging $(1.1,3,4)$, that $\langle u(t)\rangle$ is determined by the original initialboundary value problem but now with a known forcing function, the expected value of $f(x, t)$. We will henceforth take $f(x, t)$ to be of zero mean so that $u$ is also of zero mean. We can therefore concentrate on the development of a direct method for the second order response statistics of $(1.1,3.4)$ which is more efficient than any available method as far as a numerical solution of the meansquare properties of the response is concerned. By a more efficient method, we mean an improvement by at least a factor $n \times m$ in the computing time required for a numerical solution over that required by the Green's function method and its variants (e.g. the correlation function method to be described in section (3.2)). Here $m$ is the number of mesh points used in $\mathrm{V}$ and $\mathrm{n}$ is the number of time steps. The essential feature of the method is the formulation of an initial-boundary value problem for the spatial correlation matrix of the response? the counterpart of the covariance matrix of a discrete dynamical system. This spatial correlation matrix then serves as the initial condition of another initial-boundary value problem for the correlation matrix, which completely characterizes the second order statistics of the response. To bring out some of the difficulties in the formulation of the desired initialboundary value problems, the method was discussed in [5] for a scalar second order equation in both $\mathrm{x}$ and $\mathrm{t}$ with $\mathrm{x}$ itself being a scalar and with special classes of random excitations. The present paper treats the general problem defined by $(1.1,3,4)$ for a more general random $f(x, t)$ and resolves additional theoretical difficulties not encountered in [5].

While this direct method (henceforth referred to as the Spatial Correlation Method) is particularly useful for problems for which a solution by numerical integration is necessary, it is also competitive with other available methods for problems solvable by both. This is illustrated by applying the present method to the problem of a simply-supported rectangular plate driven at a point by a bandlimited white noise process solved recently in [6]. We will see that, in contrast to the analysis of [6] which involves integration in the complex plane, an exact solution of the problem by our solution scheme involves only simple algebraic calculations.

\section{Temporally uncorrelated excitations}

\subsection{The spatial correlation matrix}

To allow for some flexibility, we write the random load function $f(x, t)$ as

$$
f(x, t)=E(x, t) N(x, t)
$$

where $\mathrm{E}(\mathrm{x}, \mathrm{t})$ is a known (matrix) envelope function and $\mathrm{N}(\mathrm{x}, \mathrm{t})$ is a (vector) random process of zero mean (since $\mathbf{f}$ is assumed to be of zero mean). Henceforth, we will call $\mathrm{N}(\mathrm{x}, \mathrm{t})$ the random excitation to be distinguished from $\mathrm{f}(\mathrm{x}, \mathrm{t})$ which will be referred to as the random load. For many problems including the problem of a rotating blade advancing in atmospheric turbulence, $\mathrm{E}(\mathrm{x}, t)$ is a nonconstant continuous (matrix) function of $\mathrm{x}$ and $t$ (see equation (1.2)). 
Guided by the results of [5], we consider first the class of random excitations which is temporally uncorrelated so that

$$
R_{N}(x, t \mid y, \tau)=\left\langle N(x, t) N^{T}(y, \tau)\right\rangle=R_{S}(x, y, \tau) \delta(t-\tau)
$$

with $R_{S}^{T}(x, y, t)=R_{S}(y, x, t)$ where ()$^{T}$ is the transpose of ( ). For this class of random excitations, we have from (1 .5), (2.1) and (2.2) that

$$
\begin{aligned}
\left\langle u(x, t) f^{T}(y, t)\right\rangle & =\frac{1}{2} \int_{V} \mathrm{G}\left(\mathrm{x}, \downarrow \mid x^{\prime}, t\right) E\left(x^{\prime}, t\right) R_{S}\left(x^{\prime}, y, t\right) E^{T}(y, t) d x \\
& =\frac{1}{2} E(x, t) R_{S}(x, y, t) E^{T}(y, t)
\end{aligned}
$$

since $G\left(x, t \mid x^{\prime}, t\right)=I \delta\left(x-x^{\prime}\right)$ where 1 is the identity matrix (see appendix). Similarly, we have

$$
\left\langle f(x, t) u^{T}(y, t)\right\rangle=\frac{1}{2} E(x, t) R_{S}(x, y, t) E^{T}(y, t) .
$$

We now obtain the spatial correlation matrix $U(x, y, t)$ of the unknown vector function $\mathrm{u}$ defined by the relation

$$
U(x, y, t)=\left\langle u(x, t) u^{T}(y, t)\right\rangle .
$$

We do this by formulating an initial-boundary value problem for $U$. To get an equation for $\mathrm{U}$, we ensemble-average the identity $\left[u(x, t) u^{T}(y, t)\right]_{t}=u_{t}(x, t) u^{T}(y, t)$ $+\boldsymbol{u}(x, t) \boldsymbol{u}_{t}^{\boldsymbol{T}}(y, t)$ and use equation (1.1) to eliminate $\boldsymbol{u}_{\boldsymbol{t}}$ and $\boldsymbol{u}_{\boldsymbol{t}}^{\boldsymbol{T}}$. With the help of (2.3) and (2.4), the result can be written as

$$
U_{t}+L_{x}[U]+\left(L_{y}\left[U^{T}\right]\right)^{T}=g(x, y, t)
$$

where

$$
g(x, y, t)=E(x, t) R_{s}(x, y, t) E^{T}(y, t)=g^{T}(y, x, t)
$$

and where the subscripts $x$ and $y$ indicate that we are working in the $(x, t)$ and the $(y, t)$ space respectively.

Equation (2.6) is a matrix partial differential equation with a known right hand member. The domain of this equation is the product space $(0, \infty) \times V \times V$. To supplement this equation, we have from (1.3) the initial condition

$$
U(x, y, 0)=0, \quad(x, y) \in(\bar{V} \times \bar{V})
$$

and from (1.4) the boundary conditions for $t>0$

$$
\begin{array}{ll}
B_{x}[U(x, y, t)]=0, & (x, y) \in(S \times V) \\
B_{y}\left[U^{T}(x, y, t)\right]=0, & (x, y) \in(V \times S) .
\end{array}
$$

The solution of the initial-boundary value problem defined by $(2.6,8,9)$ can always be obtained by numerical integration in a straightforward manner. Our experience with dynamical systems [3] suggests that even if an exact solution of the problem for $U$ is not possible, we can often take advantage of the special properties of the operator $L$ to obtain an approximate solution of the problem defined by $(2.6,8,9)$ in terms of known functions by the available methods of applied mathematics. 
If $\mathrm{E}(\mathrm{x}, \mathrm{l})$ is continuous in $\mathrm{x}$ and $\boldsymbol{R}_{\boldsymbol{S}}(x, \mathrm{y}, \mathrm{t})$ is continuous in both $\mathrm{x}$ and $\mathrm{y}$, then $\mathrm{g}(\mathrm{x}, \mathrm{y}, \mathrm{t})$ is continuous in both $\mathrm{x}$ and $\mathrm{y}$. The solution $\mathrm{U}$ is therefore at least continuous in $\mathrm{x}$ and $\mathrm{y}$, in particular across the subspace $\mathrm{x}=\mathrm{y}$ of the product space $\mathrm{V} x \mathrm{~V} . \mathrm{U}(\mathrm{x}, \mathrm{x}, \mathrm{t})$ is therefore the well defined covariance matrix. If a solution by numerical integration is necessary, to obtain the same results by the Green's function method or its variants would have increased the required computing time at least by a factor $n^{2} \times m$ where $m$ is the number of mesh points in $\mathrm{V}$ and $n$ is the number of time steps. Along with the reduction in computing time, there is also a significant reduction of out-of-core storage requirement which should lead to a further reduction in machine time as well as a simplification in the programming of the machine computation.

In the special case where $L$ is a matrix $A$ with coefficients independent of $x$ and $f(x, t)=f(t)$ so that $E(x, t)=E(t)$ and $R_{S}(x, y, t)=Q(t)$, equation (2.6) reduces to

$$
U_{t}+A U+U A^{T}=E(t) Q(t) E^{T}(t)
$$

which is what we should have for the covariance matrix of a dynamical system characterized by the vector ordinary differential equation

$$
u_{t}+A(t) u=f(t)
$$

\subsection{The correlation matrix}

To get the correlation matrix of $\mathrm{u}$, defined by

$$
R_{u}(x, t ; y, \tau)=\left\langle u(x, t) u^{T}(y, \tau)\right\rangle,
$$

we postmultiply $(1.1,3,4)$ by $u^{T}(y, \tau)$ and ensemble average. The results are

$$
\begin{gathered}
{\left[R_{u}\right]_{t}+L_{x t}\left[R_{u}\right]=R_{f u}(x, t ; y, \tau), \quad(t>0, x \in V)} \\
R_{u}(x, 0 ; y, \tau)=0 \quad(x \in \bar{V}) \\
B_{x t}\left[R_{u}\right]=0 \quad(t>0, x \in S)
\end{gathered}
$$

where the subscripts $x$ and $t$ indicate that we are working in the $(x, t)$ space and where

$$
R_{f u}(x, t ; y, \tau)=\left\langle f(x, t) u^{T}(y, \tau)\right\rangle=R_{u f}^{T}(y, \tau ; x, t) .
$$

For a general $f(x, t), \boldsymbol{R}_{f u}$ is not known, and we have to determine $\boldsymbol{R}_{f u}$ by solving another initial-boundary value problem (see section (3.2)). But for a temporally uncorrelated $f(x, t)$, we have from (1.5) and (2.2)

$$
R_{f u}(x, t ; y, \tau)=H(\tau-t) E(x, t) \int_{V} R_{S}\left(x, y^{\prime}, t\right) E^{T}\left(y^{\prime}, t\right) G^{T}\left(y, \tau \mid y^{\prime}, t\right) d y^{\prime}
$$

where $H(z)$ is the unit step function. Therefore, we have $R_{f u}=0$ for all $t>\tau$ so that

$$
\left[R_{u}\right]_{t}+L_{x t}\left[R_{u}\right]=0 \quad(t>\tau, x \in V) .
$$

Moreover, as a function of $t, R_{f u}(x, t ; y, \tau)$ has at most a finite jump discontinuity across $t=\tau$ if $E$ behaves no worse than that at $t=\tau$. Therefore, $R_{u}$ is continuous across $t=\tau$. From the definition of $R_{u}$ and $U$, we have

$$
R_{u}(x, \tau ; y, \tau)=U(x, y, \tau) .
$$


But we have already obtained $U$ in section (2.1). Therefore, equations $(2.18,19,15)$ define an initial-boundary value problem for $\boldsymbol{R}_{\boldsymbol{u}}$ with $(y, \tau)$ as parameters. The solution of this problem is even easier to obtain than the spatial correlation matrix. From the point of view of numerical integration, to obtain the correlation matrix for a fixed pair of $(y, \tau)$ by the solution scheme developed here is more efficient (by essentially the same factor as cited in the last section) than the straightforward correlation function approach (see section (3.2)).

Once we have obtained $R_{u}$ for $t>\tau$, we can get $\boldsymbol{R}_{u}$ for $t<\tau$ from the symmetry condition

$$
R_{u}(x, t ; y, \tau)=R_{u}^{T}(y, \tau ; x, t)
$$

It seems appropriate at this point to emphasize that the results obtained in this and the last section are fundamental to the rest of the paper. In fact, the main objective from here on is to reduce the general problem to one with a temporally uncorrelated excitation. We will do this in several steps in sections (3), (4) and (5).

\subsection{A point-driven simply supported rectangular plate}

In spite of the rather severe restriction imposed by the requirement that the loading be temporally uncorrelated, the formulation of the last two sections is already applicable to a number of technical problems including the problem of rotating wing mentioned earlier. Also, its usefulness is not restricted to such an analytically untractable problem for which other methods are either inapplicable or impractical. To demonstrate this point, we will apply the method developed here to the problem of a simply supported rectangular elastic plate driven at a point $\left(x_{1 f}, x_{2 f}\right)$ by an ideal white noise process. The solution for a band-limited white noise excitation may be obtained by deleting from the solution for the ideal white noise case terms associated with a natural frequency higher than the cutoff frequency. An approximate solution for the band-limited white noise problem was obtained recently in [6] by the method of transfer function. Unlike the analysis of [6] which involves integration in the complex plane, an exact solution in our case involves only simple algebraic calculations.

In dimensionless form, the equation of motion for the transverse displacement of a uniform rectangular elastic plate with internal damping may be written as

$$
w_{t t}+\beta w_{t}+\nabla^{4} w=f\left(x_{1}, x_{2}, t\right) \quad\left(t>0,0<x_{1}, x_{2}<1\right)
$$

where $\beta$ is a positive constant and $\nabla^{2}()=()_{x_{1} x_{1}}+r^{2}()_{x_{2} x_{2}}$. We may take the aspect ratio of the plate, $r$, to be not greater than unity. We consider here the special case

$$
f\left(x_{1}, x_{2}, t\right)=\delta\left(x_{1}-x_{1 f}\right) \delta\left(x_{2}-x_{2 f}\right) n(t) \equiv \delta\left(x-x_{f}\right) n(t)
$$

where $n(t)$ is a white noise process so that $R_{n}(t, \tau)=\delta(t-\tau)$. The plate is initially at rest so that

$$
w\left(x_{1}, x_{2}, 0\right)=w_{t}\left(x_{1}, x_{2}, 0\right)=0 \quad\left(0 \leq x_{1}, x_{2} \leq 1\right) .
$$

The plate is simply-supported at all four edges so that we have

$$
\begin{array}{lll}
x_{1}=0,1: & w=w_{x_{1} x_{1}}=0, & \left(t>0,0 \leq x_{2} \leq 1\right) \\
x_{2}=0,1: & w=w_{x_{2} x_{2}}=0, & \left(t>0,0 \leq x_{1} \leq 1\right) .
\end{array}
$$


To get (2.20) into the form (1.1), we set $\boldsymbol{u}_{1}=\mathrm{w}$ and $\boldsymbol{u}_{\mathbf{2}}=\boldsymbol{w}_{\boldsymbol{t}}$ and write (2.20) as

$$
u_{t}+\left(\begin{array}{lr}
0 & -1 \\
\nabla^{4} & \beta
\end{array}\right) u=\left(\begin{array}{l}
0 \\
1
\end{array}\right) n(t) \delta\left(x-x_{f}\right)
$$

For this equation, the corresponding matrix equation (2.6) for the spatial correlation matrix, $U(x, Y, t)$, is equivalent to the following four scalar equations :

$$
\begin{aligned}
& {\left[U_{11}\right]_{t}-U_{21}-U_{12}=0,} \\
& {\left[U_{12}\right]_{t}-U_{22}+\beta U_{12}+\nabla_{y}^{4} U_{11}=0} \\
& {\left[U_{21}\right]_{t}-U_{22}+\beta U_{21}+\nabla_{x}^{4} U_{11}=0} \\
& {\left[U_{22}\right]_{t}+2 \beta U_{22}+\nabla_{x}^{4} U_{12}+\nabla_{y}^{4} U_{21}=\delta\left(x-x_{f}\right) \delta\left(y-x_{f}\right)}
\end{aligned}
$$

where

$$
U_{i j}=\left\langle u_{i}\left(x_{1}, x_{2}, t\right) u_{j}\left(y_{1}, y_{2}, t\right)\right\rangle
$$

are defined for $t \geq 0$ and $0 \leq x_{j}, y_{k} \leq 1$.

The initial and boundary conditions for $U$ are

$$
U(x, y, 0)=0, \quad\left(0 \leq x_{j}, y_{k} \leq 1\right)
$$

and

$$
\begin{array}{lll}
x_{1}=v, 01: & U(x, y, t)=U_{x_{1} x_{1}}(x, y, t)=0 & \left(t>0,0 \leq x_{2}, y_{k} \leq 1\right) \\
x_{2}=v, 01: & U(x, y, t)=U_{x_{2} x_{2}}(x, y, t)=0 & \left(t>0,0 \leq x_{1}, y_{k} \leq 1\right) \\
y_{1}=0,1: & U(x, y, t)=U_{y_{1} y_{1}}(x, y, t)=0 & \left(t>0,0 \leq x_{j}, y_{2} \leq 1\right) \\
y_{2}=0,1: & U(x, y, t)=U_{y_{2} y_{2}}(x, y, t)=0 & \left(\mathrm{t}>0,0 \leq x_{j}, y_{1} \leq 1\right) .
\end{array}
$$

These conditions follow from (2.22) and (2.23).

A formal solution of $(2.25,27,28)$ may be obtained by expanding $U_{\boldsymbol{i} j}$ and the forcing in double Fourier series :

$$
\begin{gathered}
\delta\left(x-x_{f}\right) \delta\left(y-x_{f}\right)=\sum_{j, k, m, n=1}^{\infty} \phi{ }_{j k}(x) \phi_{j k}\left(x_{f}\right) \phi_{m n}(y) \phi_{m n}\left(x_{f}\right) \\
U=\sum_{j, k, m, n=1}^{\infty} V_{j k m n}(t) \phi_{j k}(x) \phi_{j k}\left(x_{f}\right) \phi_{m n}(y) \phi_{m n}\left(x_{f}\right)
\end{gathered}
$$

where

$$
\phi_{p q}(z)=2 \sin \left(p \pi z_{1}\right) \sin \left(q \pi z_{2}\right)
$$

Upon substituting (2.29) and (2.30) into (2.25), we find that

$$
U=\sum_{j, k, m, n=1}^{\infty}\left[\sum_{l=1}^{4} c_{l j k m n} W_{l j k m n} e^{-} a_{l k j m n} t+W_{o j k m n}\right] \phi_{j k}(x) \phi_{j k}\left(x_{f}\right) \phi_{m n}(y) \phi_{m n}\left(x_{f}\right)
$$


where $c_{l j k m n}$ are arbitrary scalar constants,

$$
\begin{aligned}
a_{l j k m n} & =\beta \pm \frac{1}{2} i \sqrt{4 \alpha_{j k}^{2}-\beta^{2}} \pm \frac{1}{2} i \sqrt{4 \alpha_{m n}^{2}-\beta^{2}} \\
\alpha_{p q} & =(p \pi)^{2}+r^{2}(q \pi)^{2} \\
W_{o j k m n} & =\frac{1}{\Delta}\left(\begin{array}{cc}
-2 \beta & \alpha_{j k}^{2}-\alpha_{m n}^{2} \\
\alpha_{m n}^{2}-\alpha_{j k}^{2} & \beta\left(\alpha_{j k}^{2}+\alpha_{m n}^{2}\right)
\end{array}\right) \\
\Delta & =2 \beta^{2}\left(\alpha_{j k}^{2}+\alpha_{m n}^{2}\right)-\left(\alpha_{j k}^{2}-\alpha_{m n}^{2}\right)^{2}
\end{aligned}
$$

and where $W_{l j k m n}$ are both known $2 \times 2$ constant matrices which will not be written out here.

Since both the differential equations (2.25) and the boundary conditions (2.28) are satisfied by (2.32) it remains to satisfy the initial conditions. The homogeneous condition (2.27) can be satisfied by an appropriate choice of the still arbitrary constants $c_{l j k m n}$. Though they can be easily determined by way of the orthogonality condition among the $\phi$ 's, we will not be concerned with the explicit expression for these constants here. Instead, we will concentrate on the steady state solution for the covariance matrix $U(x, x ; t)$ for the purpose of comparison with the results obtained in [6].

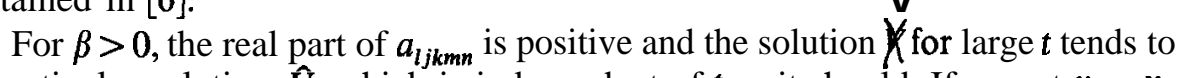
a particular solution, $\hat{U}$, which is independent of $\boldsymbol{t}$ as it should. If we set $y_{k}=x_{k}$ in this steady state (stationary) solution, we get

$$
\hat{U}(x, x, t)=\sum_{j, k, m, n=1}^{\infty} W_{o j k m n} \phi_{j k}(x) \phi_{j k}\left(x_{f}\right) \phi_{m n}(x) \phi_{m n}\left(x_{f}\right)
$$

From the expression for the matrix $W_{o j k m n}$ given in (2.33), we see that the series solution for the dimensionless steady state mean square displacement and power flow converges uniformly and absolutely. But the dimensionless steady state mean square velocity,

$$
\begin{aligned}
& \hat{U}_{22}=\frac{1}{2 \beta} \sum_{j, k=1}^{\infty} \phi_{j k}^{2}(x) \phi_{j k}^{2}\left(x_{f}\right) \\
& +\sum_{\substack{j \neq m, n \\
\text { or } k \neq m . n}}^{\infty} \frac{\beta\left(\alpha_{j k}^{2}+\alpha_{m n}^{2}\right)}{2 \beta^{2}\left(\alpha_{j k}^{2}+\alpha_{m n}^{2}\right)-\left(\alpha_{j k}^{2}-\alpha_{m n}^{2}\right)^{2}} \phi_{j k}(x) \phi_{j k}\left(x_{f}\right) \phi_{m n}(x) \phi_{m n}\left(x_{f}\right),
\end{aligned}
$$

is dominated by at least one divergent subseries. This is not surprising since we used an ideal white noise process in our concentrated forcing. Had we started with a band-limited white noise point-forcing, the corresponding series solution would have terminated after a finite number of terms. In that case, no problem with convergence would arise.

With the sum in (2.35) taken only over all integers $p$ and $q$ which satisfy the relation

$$
\alpha_{p q}=(p \pi)^{2}+r^{2}(q \pi)^{2}=k_{c}^{2} \pi^{2}
$$

where $\boldsymbol{k}_{\boldsymbol{c}}$ is the maximum wave number associated with the cutoff frequency, the first series on the right side of (2.35) is exactly what was found in [6] assuming that the contribution from the interaction of different modes can be neglected whenever the average modal overlap ratio is sufficiently small. This first series is indeed a good approximation for a small average modal overlap ratio if $r$ is 
not rational. But we see from the definition of $\alpha_{p q}$ in (2.33) that, when $r$ is rational, $\alpha_{j k}-\alpha_{m n}$ may still vanish even if $\mathrm{j} \neq m, n$ and $k \neq m, n$. For example, we have $\alpha_{18}=\alpha_{47}$ when $r=1$. Therefore, an accurate approximation for a lightly damped plate $\left(2 \beta^{2} \ll \pi^{4}\right)$ would be

$$
\begin{aligned}
\hat{O}_{22} & \equiv \frac{1}{2 \beta}\left[\sum_{j, k=1} \phi_{j k}^{2}(x) \phi_{j k}^{2}\left(x_{f}\right)\right. \\
& \left.+\sum_{\substack{\alpha_{j k}=\alpha_{m n} \\
j \neq m, n \\
k \neq m, n}} \phi_{j k}(x) \phi_{j k}\left(x_{f}\right) \phi_{m n}(x) \phi_{m n}\left(x_{f}\right)\right]
\end{aligned}
$$

instead of the first series of (2.37) alone.

On the other hand, the aspect ratio for the plate used in [6] is $\frac{11}{59}$. The leading term of the second series in (2.37) is associated with a frequency of about $17,000 \mathrm{~Hz}$ for the particular aluminum plate of $\frac{1}{16}$ inch thickness. Since the wide band stationary excitation for the experiment in [6] is nominally flat to only about $5,000 \mathrm{~Hz}$ with an appreciable roll-off occurring at $6,000 \mathrm{~Hz}$, the first series of (2.37) is in fact adequate for the analysis of [6].

\section{Temporally random excitations}

If $\mathrm{N}(\mathrm{x}, \mathrm{t})$ is temporally correlated so that (2.2) is not satisfied, the solution scheme developed in section (2) is no longer directly applicable. It will be applicable if we can somehow reduce the problem to one with a temporally uncorrelated random excitation. In this section, we show that such a reduction is possible for the class of temporally correlated random excitations which are independent of the spatial coordinates so that $\mathrm{N}(\mathrm{x}, \mathrm{t})=\mathrm{N}(\mathrm{t})$ and $R_{N}(x, t ; y, \tau)=R_{N}(t, \tau)$. The random excitation of the rotating blade problem is often assumed to belong to this class. We see from that problem that the random load $f(x, t)$ itself may still be a function of $x$ through the envelope function $E(x, t)$.

For this class of random excitations, we can associate $\mathrm{N}(\mathrm{t})$ with the response of a (fictitious) linear dynamical system to a shot noise (i.e., delta-correlated $n(t)$ ). By this, we mean that $\mathrm{N}(\mathrm{t})$ and the output of the supplementary linear system have the same first and second order statistics. By setting up stationary or nonstationary shaping filters of first or higher order with white noise input, almost any correlation function for $\mathrm{N}(\mathrm{t})$ can be approximated well enough for practical purpose $[7,8]$.

\subsection{The spatial correlation matrix}

For the purpose of obtaining the second order statistics of $u$, we will think of $\mathrm{N}(\mathrm{t})$ as the steady state response of a linear dynamical system characterized by the equation

$$
N_{t}+A(t) N=F(t) n(t)
$$

where $A$ and $F$ are known matrix functions of $\mathrm{t}$ and $n(t)$ is a vector shot noise process with zero mean, i.e.

$$
\langle n(t)\rangle=0, \quad\left\langle n(t) n^{T}(\tau)\right\rangle=Q(\tau) \delta(t-\tau) .
$$


By using (3.1), we essentially assume that a component of the vector $\mathrm{N}(\mathrm{t})$ is the response to shot noise of a filter of order not higher than $l$ where $l$ is the dimension of $\mathrm{N}(\mathrm{t})$ itself. If this condition is not satisfied by $\mathrm{N}(\mathrm{t})$, then $\mathrm{N}(\mathrm{t})$, instead of being uncorrelated, should itself be taken as the response of another dynamical system of order no higher than 2 to shot noise, and so on. We will consider in what follows the case where a single equation of the form (3.1) suffices since no new element is introduced to our method of solution by additional equations.

With $\mathrm{N}(\mathrm{t})$ treated as an unknown, the two equations (3.1) and (1.1) form a larger system of dimension $2 l$ of the form (1.1) but now with a temporally uncorrelated excitation. In principle, we can now apply the solution scheme developed in section (2) to solve this problem once we have formulated the appropriate initial and boundary conditions for the new system. But since the new system may be very large (especially if filters of an order higher than $l$ are needed to relate $\mathrm{N}(\mathrm{t})$ to a temporally uncorrelated process), it may be worth noting the following less direct but more efficient method of solution.

The solution of (3.1) can be expressed in terms of the associated impulse response function $\mathrm{h}(\mathrm{t}, \mathrm{z})$ as

$$
N(t)=\int_{-\infty}^{t} h(t, z) F(z) n(z) d z
$$

from which follow the relations

$$
\begin{aligned}
& \left\langle N(t) n^{T}\left(t^{\prime}\right)\right\rangle=\int_{=a l}^{t} h(t, z) F(z) Q\left(t^{\prime}\right) \delta\left(z-t^{\prime}\right) d z=H\left(t-t^{\prime}\right) h\left(t, t^{\prime}\right) F\left(t^{\prime}\right) Q\left(t^{\prime}\right) \\
& \left\langle n(t) N^{T}\left(t^{\prime}\right)\right\rangle=H\left(t^{\prime}-t\right) Q(t) F^{T}(t) h^{T}\left(t^{\prime}, t\right)
\end{aligned}
$$

With (3.4) we get from (1.5)

$$
\left\langle n(t) u^{T}(x, t)\right)=\left\langle u(x, t) n^{T}(t)\right)=0
$$

Now we form the ensemble average of the relation $\left(u N^{T}\right)_{t}=u_{t} N^{T}+u N_{t}^{T}$ and use (1.1) and (3.1) to get

$$
P_{\imath}+L[P]+P A^{T}=E(x, t) R_{N}(t, t)
$$

where

$$
P(x, t)=\left\langle u(x, t) N^{T}(t)\right\rangle
$$

Equation (3.5) is supplemented by the initial condition

$$
P(x, 0)=0 \quad(x \in \bar{V})
$$

and the boundary condition

$$
B[P]=0 \quad(t>0, x \in S)
$$

which follow from (1.3) and (1.4), respectively. Equations $(3.6,8,9)$ determine $\mathrm{P}(\mathrm{x}, \mathrm{t})$.

We now repeat the derivation which led to (2.6) but keep in mind that the relations (2.3) and (2.4) do not hold for temporally correlated excitations. The result is

$$
U_{t}+L_{x}[U]+\left(L_{y}\left[U^{T}\right]\right)^{T}=P(x, t) E^{T}(y, t)+E(x, t) P^{T}(y, t) .
$$


The initial and boundary conditions for the spatial correlation matrix remain as given by (2.8) and (2.9).

The solution scheme formulated here is more complicated than the special case of a temporally uncorrelated excitation where we do not have to solve for $\mathrm{P}(\mathrm{x}, \mathrm{t})$. But from the point of view of a numerical solution, the magnitude of the computational effort remains unchanged. We emphasize that it is not necessary to store the solution for $\mathrm{P}$ generated by $(3.6,8,9)$ to be used in $(3.10)$. At any particular time step $\boldsymbol{t}_{\boldsymbol{n}}$, we can use $\boldsymbol{P}_{\boldsymbol{n}}$ to calculate $\boldsymbol{P}_{\boldsymbol{n}+\mathbf{1}}$ as well as $\boldsymbol{U}_{\boldsymbol{n}+\mathbf{1}}$. There is no need to retain $P_{n}$ in core after that.

\subsection{The correlation matrix}

To obtain the correlation matrix $R_{u}(x, t ; y, \tau)$ as defined by $(2.12)$, we observe that for a temporally correlated excitation, the important relation (2.17) no longer holds. Therefore, we can not use the simple scheme described in section (2.2) to get the correlation matrix.

A straightforward procedure at this point would be to get another equation for $\boldsymbol{R}_{f u}(\boldsymbol{x}, t ; y, \tau)$ as defined by (2.16). This can be done by pre-multiplying the transpose of $(1.1)$ by $f(y, \tau)$ and ensemble average. After we interchange the role of $(x, t)$ and $(y, \tau)$ in the result, we get

$$
\left[R_{f u}\right]_{\tau}+\left(L_{y t}\left[R_{f u}^{T}\right]\right)^{T}=R_{f}(x, t ; y, \tau)=E(x, t) R_{N}(t, \tau) E^{T}(y, \tau)
$$

where $R_{f}$ and $R_{N}$ are the correlation matrix off and $N$, respectively. Similarly, we get from (1.3) and (1.4)

$$
\begin{aligned}
R_{f u}(x, t ; y, 0) & =0 \\
B_{y \tau}\left[R_{f u}^{T}(x, t ; y, \tau)\right]=0 & (y \in V) \\
& (\tau>0, y \in S) .
\end{aligned}
$$

Since $R_{f}$ is known, we can solve (3.11-13) for $R_{f u}$ with $x$ and $t$ as parameters. The result is then used in (2.13-15) to determine $R_{u}(x, t ; y, \tau)$ as a function of $x$ and $t$ with y and $\tau$ as parameters. This method of solution is what has been referred to as the correlation function method in this paper and discussed previously in $[9,5]$ and elsewhere.

While this solution scheme is undoubtedly a possible scheme it is not a practical one if the relevant initial-boundary value problems are to be solved by numerical integration [5]. A more efficient solution scheme is to combine (1.1) and (3.1) as one big system*

$$
v_{t}+L^{*}[v]=E^{*}(t) N^{*}(t)
$$

where

$$
v=\left(\begin{array}{l}
u \\
N
\end{array}\right), L *=\left(\begin{array}{cc}
L & -E(x, t) \\
0 & A(t)
\end{array}\right), E *=\left(\begin{array}{cc}
0 & 0 \\
0 & F(t)
\end{array}\right)
$$

and where $\mathrm{N}^{*}(\mathrm{t})$ is a $2 l$ dimensional vector shot noise process of zero mean with $R_{N^{*}}\left(t, t^{\prime}\right)=Q^{*}\left(t^{\prime}\right) \delta\left(t-t^{\prime}\right)$. Equation (3.14) is again of the form (1.1) but now the excitation is temporally uncorrelated. We can therefore make use of the result of section (2.2) to calculate the correlation matrix for $v$, a submatrix of which is the correlation matrix of $u$.

* We again assume that no filter of order higher than the dimension of $\mathrm{N}(\mathrm{t})$ is required. 
According to the result of section (2.2), we have for the correlation function $R_{v}(x, t ; y, \tau)$ the equation

$$
\left[R_{v}\right]_{t}+L_{x t}^{*}\left[R_{v}\right]=0 \quad(t>\tau)
$$

with

$$
R_{v}(x, t ; y, \tau)=\left(\begin{array}{ll}
R_{u}(x, t ; y, \tau) & R_{u N}(x, t ; y, \tau) \\
R_{N u}(x, t ; y, \tau) & R_{N}(x, t ; y, \tau)
\end{array}\right) .
$$

Since $N(t)$ does not depend on $x$, we have

$$
\begin{aligned}
R_{u N}(x, t ; y, \tau) & =\left\langle u(x, t) N^{T}(\tau)\right\rangle=R_{u N}(x, t ; \tau) \\
R_{N u}(x, t ; y, \tau) & =\left\langle N(t) u^{T}(y, \tau)\right\rangle=R_{N u}(t ; y, \tau) \\
R_{N}(x, t ; y, \tau) & =\left\langle N(t) N^{T}(\tau)\right\rangle=R_{N}(t, \tau) .
\end{aligned}
$$

While we can now obtain the appropriate initial and boundary conditions for $\boldsymbol{R}_{\boldsymbol{v}}$ to complete the formulation of the initial-boundary value problem, it is only consistent with our effort to obtain an efficient algorithm to make the following observation.

The matrix equation (3.16) is equivalent to four matrix equations for the four submatrices $\boldsymbol{R}_{u}, \boldsymbol{R}_{\mathrm{Nu}}, \boldsymbol{R}_{\mathrm{uN}}$ and $\boldsymbol{R}_{\boldsymbol{N}}$. Of these equations, only two are of interest to us here. They are

$$
\left[R_{N u}\right]_{t}+A(t) R_{N u}=0, \quad(t>\tau)
$$

and

$$
\left[R_{u}\right]_{t}+L_{x t}\left[R_{u}\right]=E(x, t) R_{N u} \quad(t>\tau) .
$$

The first equation is an ordinary differential equation in $\boldsymbol{t}$ for $\boldsymbol{R}_{\boldsymbol{N} u}$ with y and $\tau$ as parameters. The initial condition for this equation is

$$
R_{N u}(\tau ; y, \tau)=P^{T}(y, \tau)
$$

which follows from the definition of the two quantities involved. Note that $P^{T}(y, \tau)$ has already been obtained in section (3.1).

The solution of (3.19) and (3.21) is then used in (3.20), supplemented by the initial condition

$$
R_{u}(x, \tau ; y, \tau)=U(x, y, \tau) \quad(x \in \bar{V})
$$

and the boundary condition

$$
B_{x t}\left[R_{u}(x, t ; y, \tau)\right]=0 \quad(t>\tau, x \in S) .
$$

From the point of view of a numerical solution, it is more efficient to solve the two problems for $R_{u}$ and $R_{N u}$ simultaneously. At any time step $t_{n}, R_{N u}\left(t_{n} ; y, \tau\right)$ is used for the determination of both $R_{N u}\left(t_{n+1} ; y, \tau\right)$ and $R_{u}\left(x, t_{n+1} ; y, \tau\right)$. There is no need to keep $R_{N u}\left(t_{n} ; y, \tau\right)$ after that.

\section{Excitations with a separable correlation matrix}

For many technical problems, the correlation function of the random excitation $\mathrm{N}(\mathrm{x}, \mathrm{t})$ is the product of a function in the spatial variables and one in the time 
variables,

$$
R_{N}(x, t ; y, \tau)=R_{S}(x, y) R_{T}(t, \tau) .
$$

or, more generally, a finite sum of such products. This is true for instance in the case of a random pressure field associated with boundary layer turbulence on a flat plate (if we use a moving coordinate in the direction of the flow velocity [1]). Note that neither the load $\mathrm{f}(\mathrm{x}, \mathrm{t})$ nor its correlation function is required to be separable. For the special case of $R_{T}(t, \tau)=Q(\tau) \delta(t-\tau)$, the problem was solved in section (2). We now solve the more general case (4.1) by reducing it to this special case.

The reduction is similar to that of section (3) where we treated the corresponding problem with $\mathrm{N}(\mathrm{x}, \mathrm{t})$ independent of $\mathrm{x}$. Instead of associating $N(x, \mathrm{t})$ with the response of a linear dynamical system to shot noise, we now associate it with the response to a random excitation $\mathrm{n}(\mathrm{x}, \mathrm{t})$ which is temporally uncorrelated. More specifically, $\mathrm{n}(\mathrm{x}, \mathrm{t})$ is a process with a correlation matrix

$$
R_{n}(x, t ; y, \tau)=R_{S}(x, y) Q(\tau) \delta(t-\tau)
$$

and $N(x, t)$ is the response of some linear dynamical system to the process $n(x, t)$. That is, $\mathrm{N}(\mathrm{x}, \mathrm{t})$ is the solution of the equation

$$
N_{t}+A(t) N=F(t) n(x, t)
$$

if the supplementary dynamical system is of order not higher than the dimension of $\mathrm{N}(\mathrm{x}, \mathrm{t})$. Note that

$$
\begin{aligned}
R_{N}(x, t ; y, \tau) & =\int_{-\infty}^{l} \int_{-\infty}^{\tau} h\left(t, t^{\prime}\right) F\left(t^{\prime}\right) R_{n}\left(\dot{x}, t^{\prime} ; y, \tau^{\prime}\right) F^{T}\left(\tau^{\prime}\right) h^{T}\left(\tau, \tau^{\prime}\right) d \tau^{\prime} d t^{\prime} \\
& =R_{S}(x, y) \int_{-\infty}^{T} h\left(t, t^{\prime}\right) F\left(t^{\prime}\right) Q\left(t^{\prime}\right) F^{T}\left(t^{\prime}\right) h^{T}\left(\tau, t^{\prime}\right) d t^{\prime}
\end{aligned}
$$

where $T$ is the minimum of $t$ and $\tau$. If higher order filters are needed, then instead of requiring $\mathrm{n}(\mathrm{x}, \mathrm{t})$ to satisfy (4.2), we take it to be the response of another dynamical system to an input $\hat{n}(x, t)$ with $R_{\hat{n}}(x, t ; y, \tau)=R_{S}(x, y) Q(\tau) \delta(t-\tau)$, etc. It is not difficult to see from (4.4) that the separability of $R_{N}(x, t ; y, \tau)$ is not affected by the order of the supplementary dynamical system. For the purpose of our discussion here, it suffices to consider the case where the process $n(x, t)$ in (4.3) is temporally uncorrelated.

We can now write (4.3) and (1.1) as a single system of the form (1.1) with the loading being temporally uncorrelated. Such a system has been treated already in section (2).

Instead of dealing with this enlarged system directly, we follow the development of section (3.1) to formulate an efficient solution scheme. It is only a matter of repeating the analysis of that section to find that $\mathrm{P}(\mathrm{x}, \mathrm{y}, \mathrm{t})=\left\langle u(x, t) N^{T}(y, t)\right)$ is the solution of

$$
P_{t}+L_{x}[P]+P A^{T}=E(x, t) R_{S}(x, y) R_{T}(t, t)
$$

with

$$
\begin{aligned}
& P(x, y, 0)=0 \quad(x \in V) \\
& B_{x}[P]=0 \quad(t>0, x \in S) .
\end{aligned}
$$

In this problem, y appears only as a parameter. 
The spatial correlation matrix $U(x, y, t)$ can then be shown to be the solution of

$$
U_{t}+L_{x}[U]+\left(L_{y}\left[U^{T}\right]\right)^{T}=P(x, y, t) E^{T}(y, t)+E(x, t) P^{T}(y, x, t)
$$

with (2.8) and (2.9) as the initial and boundary conditions, respectively.

Repeating the analysis of section (3.2), we find that the cross correlation matrix $R_{N u}(x, t ; y, \tau)=\left\langle N(x, t) u^{T}(y, \tau)\right\rangle$ is the solution of

with

$$
\left[R_{N u}\right]_{t}+A(t) R_{N u}=0 \quad(t>\tau)
$$

$$
R_{N u}(x, \tau ; y, \tau)=P^{T}(y, x, \tau) \quad(x \in \bar{V}) .
$$

We find also that the correlation matrix $R_{u}(x, t ; y, \tau)$ is the solution of

$$
\begin{gathered}
{\left[R_{u}\right]_{t}+L_{x t}\left[R_{u}\right]=E(x, t) R_{N u}(x, t ; y, \tau) \quad(t>\tau, x \in V)} \\
R_{u}(x, \tau ; y, \tau)=U(x, y, \tau) \quad(x \in \bar{V}) \\
B_{x t}\left[R_{u}\right]=0 \quad(t>\tau, x \in S) .
\end{gathered}
$$

Insofar as the numerical solutions of these initial-boundary value problems are concerned, the remarks at the end of section (3.1) and (3.2) again apply.

Finally, it should be mentioned that the determination of the supplementary linear dynamical system is no more difficult for the present case than the case of $\mathrm{N}(\mathrm{x}, \mathrm{t})$ being independent of $\mathrm{x}$ discussed in section (3.1). In view of the relation (4.4), all we have to do is to find a system whose response to a shot noise process $\mathrm{n}(\mathrm{t})$ has $R_{T}(t, \tau)$ as its correlation matrix.

\section{General random excitations}

If the correlation matrix $R_{N}(x, t ; y, \tau)$ of the rand.om excitation $\mathrm{N}(\mathrm{x}, \mathrm{t})$ is neither temporally uncorrelated nor separable in space and time, none of the results obtained so far is directl y applicable. In this section, we will discuss briefly how the problem for a general random excitation can be red uced to one for an excitation with a separable correlation matrix.

Recall that the differential equation for the spatial correlation matrix $U(x, y, t)$ of the unknown $\mathrm{u}$ is

$$
U_{t}+L_{x}[U]+\left(L_{y}\left[U^{T}\right]\right)^{T}=E(x, t) P^{T}(y, x, t)+P(x, y, t) E^{T}(y, t)
$$

where

$$
\mathrm{P}(\mathrm{x}, \mathrm{y}, \mathrm{t})=\left\langle u(x, t) N^{T}(y, t)\right\rangle .
$$

This equation holds for a general random excitation $\mathrm{N}(\mathrm{x}, \mathrm{t})$. The task is to determine $P(x, y, t)$ efficiently for a random excitation which is not separable.

By (1.5) we have

$$
P(x, y, t)=\int_{V} \int_{0}^{t} G\left(x, t \mid x^{\prime}, t^{\prime}\right) E\left(x^{\prime}, t^{\prime}\right) R_{N}\left(x^{\prime}, t^{\prime} ; y, t\right) d t^{\prime} d x^{\prime} .
$$

Now, $\boldsymbol{R}_{\boldsymbol{N}}$ is a known function. If $\boldsymbol{R}_{N}$ satisfies some mild condition such as piecewise differentiability (or weaker), we can expand it as a series of the form

$$
R_{N}\left(x^{\prime}, t^{\prime} ; y, t\right)=\sum_{k=1}^{\infty} R_{S k}\left(x^{\prime}, y\right) R_{T k}\left(t^{\prime}, t\right)
$$


where $\left\{\boldsymbol{R}_{\mathbf{S k}}\left(z_{1}, z_{2}\right)\right\}$ is a complete set of orthogonal functions defined in the product space $V \times V$. Depending on the geometry of $V$ and the boundary condition (1.4), we may take $\left\{R_{S k}\right\}$ to be the set of trigonometric functions, polynomials or even the set of eigenfunctions associated with the operator $L$ (if it exists and is complete).

With (5.4), we may write (5.3) as

$$
P(x, y, t)=\sum_{k=1}^{\infty} P_{k}(x, y, t)
$$

where

$$
P_{k}(x, y, t)=\int_{V} \int_{0}^{t} G\left(x, t \mid x^{\prime}, t^{\prime}\right) E\left(x^{\prime}, t^{\prime}\right) R_{S k}\left(x^{\prime}, y\right) R_{T k}\left(t^{\prime}, t\right) \mathrm{d} t^{\prime} d x^{\prime} .
$$

If we now identify the product $R_{S k}\left(x^{\prime}, y\right) R_{T k}\left(t^{\prime}, t\right)$ as the correlation of some zero mean random process $N_{k}(x, t)$ and write $u_{k}(x, t)$ as the solution of $(1.1,3,4)$ with $\mathrm{f}(\mathrm{x}, \mathrm{t})=E(x, t) N_{k}(x, \mathrm{t})$, i.e.

$$
\begin{gathered}
{\left[u_{k}\right]_{t}+L\left[u_{k}\right]=E(x, t) N_{k}(x, t) \quad(t>0, x \in V)} \\
u_{k}(x, 0)=0 \quad(x \in \bar{V}) \\
B\left[u_{k}\right]=0 \quad(t>0, x \in S),
\end{gathered}
$$

then the problem for $u_{k}$ is one for a random excitation with a separable correlation matrix.

For the purpose of our discussion, let $\left\{N_{k}(x, t)\right\}$ be an orthogonal set, i.e. $\left\langle N_{k}(x, t) N_{j}(y, \tau)\right\rangle=0$ for all $j \neq k$. Then $\left\{u_{k}(x, \mathrm{t})\right\}$ is also an orthogonal set and

$$
U(x, y, t)=\sum_{k=1}^{\infty} U_{k}(x, y, t)
$$

where

$$
U_{k}(x, y, t)=\left\langle u_{k}(x, t) u_{k}^{T}(y, t)\right\rangle .
$$

But $U_{k}(x, y, \mathrm{t})$ is the spatial correlation matrix associated with the response $u_{k}$, and the initial-boundary value problem for $u_{k}$ is one with a separable excitationcorrelation matrix. This we know how to solve.

While we have succeeded in obtaining the spatial correlation matrix of the response for a general random excitation, it is rather unsatisfying to have to solve an infinite number of initial-boundary value problems (uncoupled to be sure) each for a random excitation with a separable correlation function. But the situation may well be that (1) the excitation-correlation function is unseparable but is a sum of a finite number of separable terms, or (2) only a small number of the $N_{k}$ 's contributes significantly to the final solution. Moreover, in view of the factor $\mathrm{n} \mathrm{x} \mathrm{m}$ in computing time mentioned earlier, we could afford to solve for quite a few $U_{k}$ without being less efficient than the correlation function method or the Green's function method.

For the sake of completeness, we note that since $\left\{N_{k}\right\}$ is an orthogonal set, we have also

$$
R_{u}(x, t ; y, \tau)=\sum_{k=1}^{\infty} R_{u_{k}}(x, t ; y, \tau)
$$


and we know how to compute $\boldsymbol{R}_{u_{\mathrm{k}}}$ for each k since the problem involves only a random excitation with a separable correlation function.

Finally, it should be mentioned that the known load-correlation function may happen to be the correlation function of the solution for another initial-boundary value problem with a temporally uncorrelated random excitation or with an excitation whose correlation function is separable. In that case, we have in effect a problem already solved in sections (2), (3) or (4) for an enlarged system. Unfortunately, there does not seem to be a systematic way for finding the needed supplementary linear continuous system in all cases.

\section{On the discretization of the continuous problem}

The merit of the Spatial Correlation Method developed herein evidently lies mainly in its efficiency for a numerical solution of the mean square properties of the response. As long as a finite difference solution of the spatial correlation function(s) is necessary, we could have just as well discretized the original problem $(1.1,3,4)$ in space and then calculated the covariance matrix and the correlation matrix for the resulting multi-degree dynamical system in a manner similar to what was done in [4]. Why then do we need the effectively equivalent Spatial Correlation Method? This important question is answered in [10] where the Spatial Correlation Method is applied to a string model of the lifting rotor blade problem.

Briefly, we see from the development in [10] that the spatial correlation approach allows us to readily analyze the stability (and therefore the convergence) of the numerical scheme employed. For the spatially one dimensional problem treated in [10] for example, we see immediately from the Spatial Correlation Method that a straight forward explicit numerical integration scheme requires $\Delta t=0\left(\Delta x^{2}\right)$ for numerical stability where $\Delta t$ is the dimensionless time step and Ax is the mesh size of the dimensionless spatial variable. We should arrive at the same conclusion in the discrete model approach, but probably only after some costly numerical experimentation.

More importantly, the specific form of the equations governing the spatial correlation functions(s) often suggests a particular numerical scheme which further reduces the computing time required by any one of the standard schemes. For the problem in [10] the spatial correlation formulation suggests almost immediately a modified explicit scheme which allows $\Delta t=O(\Delta x)$, and thereby reduces the computing time by an order of magnitude. It is not likely that we would have discovered the modified explicit scheme had we discretized the continuous problem $\mathrm{ab}$ initio.

\section{Appendix}

The Green's function $G\left(x, I \mid x^{\prime}, t^{\prime}\right)$ associated with the problem $(1.1,3,4)$ is the solution of the equation

$$
G_{t}+\mathrm{L}[\mathrm{G}]=I \delta\left(x-x^{\prime}\right) \delta\left(t-\mathrm{t}^{\prime}\right)
$$

for some point $x^{\prime}$ in $V$ and some $t^{\prime}$, subject to the causality condition

$$
G\left(x, t \mid x^{\prime}, t^{\prime}\right)=0 \quad\left(t<t^{\prime}\right)
$$


and the boundary condition

$$
\mathrm{B}[\mathrm{G}]=0 \quad\left(\mathrm{t}>\boldsymbol{t}^{\prime}, x \in \mathrm{S}\right) .
$$

We are interested here in the behavior of $\mathrm{G}$ at $t=t^{\prime}$. In particular, we want to show that

$$
G\left(x, t^{\prime}+\mid x^{\prime}, t^{\prime}\right)=I \delta\left(x-x^{\prime}\right) .
$$

To prove (A.4), we consider a function $\mathrm{W}(\mathrm{x}, \mathrm{t})$ which is the solution of

$$
W_{t}+L[W]=0 \quad\left(t>t^{\prime}\right)
$$

subject to the initial condition

$$
W\left(x, t^{\prime}+\right)=I \delta\left(x-x^{\prime}\right) \quad(x \in \bar{V})
$$

and the boundary condition

$$
\mathrm{B}[\mathrm{W}]=0 \quad\left(\mathrm{t}>t^{\prime}, x \in S\right) .
$$

We now claim that the Green's function $\mathbf{G}\left(\mathbf{x}, \mathbf{t} \mid x^{\prime}, t^{\prime}\right)$ can be written as

$$
G\left(x, t \mid x^{\prime}, t^{\prime}\right)=\mathrm{H}\left(\mathrm{t}-t^{\prime}\right) W(x, t) .
$$

As given by (A.8), G evidently satisfies the causality condition (A.2) and the boundary conditions (A.3). It remains to show that it also satisfies the differential equation (A.1). But

$$
\begin{aligned}
G_{t}+L[G] & =\mathrm{H}\left(\mathrm{t}-t^{\prime}\right)\left(W_{t}+L[W]\right)+\delta\left(t-t^{\prime}\right) W(x, t) \\
& =\delta\left(t-t^{\prime}\right) W\left(x, t^{\prime}+\right)=\delta\left(t-t^{\prime}\right) \delta\left(x-x^{\prime}\right) I
\end{aligned}
$$

where use has been made of (A.5) and (A.6). The useful relation (A.4) now follows from (A.8) and (A.6).

\section{Acknowledgement}

The research was partly supported by AROD through the Army Materials and Mechanics Research Center at Watertown, Massachusetts.

\section{References}

1. Y. K. Liv, Probabilistic theory of structural dynamics, McGraw-Hill, 1967.

2. V. V. Bolotin, Statistical methods in structural mechanics, Holden-Day, 1969.

3. F. Y. M. WAN, "Nonstationary response of linear time-varying dynamical systems to random excitation," J. Appl. Mech., 40, 1973,422-428.

4. F. Y. W AN AND C. LAKSHMIKANTHAM, "Rotor blade response to random load: a direct time domain approach," Presented at AIAA 10th Aerospace Science Meeting, Jan. 1972 (San Diego), AIAA J. 11, 1973, pp. 24-28.

5. F. Y. M. WAN, "Linear partial differential equations with random forcing," Studies in Applied Mathematics 51, 1972, pp. 163-179.

6. S. H. CRandall andL. E. WITTIG “Chladni's patterns for random vibration of a plate,” Proc. Symp. Dynamic Response of Structures (ed. G. Herrmann and N. Perrone), Pergamon, 1972, pp. 55-7 1.

7. H. L. Van TreEs, Detection, estimation and modulation theory, part I, John Wiley and Sons, 1968 (pp. 5 16-526). 
8. B. D. 0. ANDerson, J. B. MOORE and S. G. LOO, "Spectral factorization of time varying covariance functions," IEEE Trans. on Information Theory 15, 1969, pp. 550-557.

9. V. V. BOLOTIN Applications of methods of the theory of probability and the theory of reliability in calculations of structures, Publishing Co. of Literatures in Buildings, Moscow, 1971 (in Russian).

10. F. Y. M. WAN AND C. LAKSHMIKANTHAM, "The spatial correlation method and a time-varying flexible structure," to appear. (An abbreviated version of this paper was presented at the AIAA-ASME Structural Dynamics and Material Conf., Williamsburg, March, 1973 ; Paper No. 73-406.)

MASSACHUSETTS Institute of TechNology

(Received May 17, 1973) 